\title{
Permanence, Periodicity and Extinction of a Delayed Biological System with Stage-Structured Preference for Predator
}

\author{
Limin Zhang1,2*, Chaofeng Zhang1 \\ ${ }^{1}$ School of Mathematics and Finance-Economics, Sichuan University of Arts and Science, Dazhou, China \\ ${ }^{2}$ School of Mathematics, Sichuan University, Chengdu, China

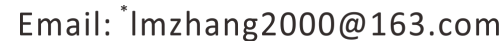

Received 27 January 2016; accepted 26 March 2016; published 29 March 2016

Copyright (C) 2016 by authors and Scientific Research Publishing Inc.

This work is licensed under the Creative Commons Attribution International License (CC BY).

http://creativecommons.org/licenses/by/4.0/

cc) (i) Open Access

\begin{abstract}
This study considers a delayed biological system of predator-prey interactions where the predator has stage-structured preference. It is assumed that the prey population has two stages: immature and mature. The predator population has different preference for the stage-structured prey. This type of behavior has been reported in Asecodes hispinarum and Microplitis mediator. By some lemmas and methods of delay differential equation, the conditions for the permanence, existence of positive periodic solution and extinction of the system are obtained. Numerical simulations are presented that illustrate the analytical results as well as demonstrate certain biological phenomena. In particular, overcrowding of the predator does not affect the persistence of the system, but our numerical simulations suggest that overcrowding reduces the density of the predator. Under the assumption that immature prey is easier to capture, our simulations suggest that the predator's preference for immature prey increases the predator density.
\end{abstract}

\section{Keywords}

Stage-Structured Preference, Permanence, Periodic Solution, Extinction, Time Delay

\section{Introduction}

In recent years, much attention has been paid to biological systems with stage structure [1]-[23]. One important ${ }^{*}$ Corresponding author.

How to cite this paper: Zhang, L.M. and Zhang, C.F. (2016) Permanence, Periodicity and Extinction of a Delayed Biological System with Stage-Structured Preference for Predator. Journal of Applied Mathematics and Physics, 4, 546-560. 
reason is that there are many species whose individual members have a life history taking them through two stages, immature and mature. Thus considering stage structure in population corresponds with the natural phenomenon. Another reason is that stage-structured ecological models are much simpler than the models governed by partial differential equations but they can exhibit phenomena similar to those of partial differential equations and many important physiological parameters can be incorporated [24]. The other reason is that the biological dynamics has long been and will continue to be one of the dominant themes in both ecology and mathematical ecology due to its universal existence and importance [25].

In References [1]-[5], the authors have studied the stability of a class of stage-structured predator-prey systems. The authors in [6] [7] have made Hopf bifurcation analysis in delayed predator-prey systems with stage structure. As we know, environmental and biological parameters (such as the seasonal effects of weather, food supplies, and mating habits) fluctuate naturally over time; thus the effects of periodically varying environments are considered to be important selective forces in systems with fluctuating environments [19] [20]. Thus, incorporating periodicity into models of stage-structured biological systems is more realistic with a changing environment. Therefore, many researchers have studied a class of periodic nonautonomous biological systems with stage structures [8]-[16] [21]. Recently, Cui and Song [21] considered the following predator-prey system with stage-structured prey:

$$
\begin{aligned}
& \dot{x}_{1}(t)=a(t) x_{2}(t)-b(t) x_{1}(t)-d(t) x_{1}^{2}(t)-p(t) x_{1}(t) y(t), \\
& \dot{x}_{2}(t)=c(t) x_{1}(t)-f(t) x_{2}^{2}(t), \\
& \dot{y}(t)=y(t)\left(-g(t)-h(t) x_{1}(t)-q(t) y(t)\right),
\end{aligned}
$$

where $a(t), b(t), d(t), p(t), c(t), f(t), g(t), h(t)$ and $q(t)$ are all continuous positive $T$-periodic functions, and $x_{1}(t), x_{2}(t)$, and $y(t)$ denote the densities of immature prey, mature prey, and predator species, respectively. They obtained a set of sufficient and necessary conditions that guarantee the permanence of the system.

In the natural world, many predators switch to alternative prey when their favored food is in short supply [22]-[24]. For example, the lynx switches to red squirrel when the snowshoe hare is scarce [25]. Even if there is only one prey type, the degree of predation or the quality (including palatability) of prey is likely to vary with its stage structure, which is likely to affect the predator's preference for different stage-structured prey. This type of behavior has been reported in Asecodes hispinarum [26], who parasitizes all 5 instars of Brontispa Logissina, but prefers to parasitize the 2nd and 3rd instars when it is exposed to all the instars of larvae, and in Microplitis mediator [27], who prefers to parasitize the 2nd and 3rd instars of Mythimna separate.

However, previous studies on prey age preference only have been done in laboratory tests. Few researchers have investigated the phenomenon with mathematical models and carried out theoretical analysis together with numerical simulation. To extend research in this area, and based on the recent study by Cui and Song [21], we consider a periodic predator-prey system with time delay and a predator with stage-structured preference.

\section{Formulation of the Model}

Let $x_{1}(t), x_{2}(t)$ and $y(t)$ represent the density of immature prey, mature prey and predator species, respectively. Our periodic predator-prey system with time delay and stage-structured preference of the predator can be described as following:

$$
\begin{aligned}
\dot{x}_{1}(t)= & b(t) x_{2}(t)-d_{1}(t) x_{1}(t)-b\left(t-\tau_{1}\right) \mathrm{e}^{-\int_{t-\tau_{1}}^{t} d_{1}(s) d s} x_{2}\left(t-\tau_{1}\right) \\
& -f_{1}(t) \frac{c_{1}(t) x_{1}(t) y(t)}{\alpha(t)+\beta(t) x_{1}(t)+\gamma(t) y(t)}, \\
\dot{x}_{2}(t)= & b\left(t-\tau_{1}\right) \mathrm{e}^{-\int_{t-\tau_{1}}^{t} d_{1}(s) \mathrm{d} s} x_{2}\left(t-\tau_{1}\right)-d_{2}(t) x_{2}^{2}(t)-f_{2}(t) \frac{h_{1}(t) x_{2}^{2}(t) y(t)}{e(t)+x_{2}^{2}(t)}, \\
\dot{y}(t)= & y(t)\left(-d_{3}(t)+\tilde{f}_{1}(t) \frac{c_{2}(t) x_{1}\left(t-\tau_{2}\right)}{\alpha(t)+\beta(t) x_{1}\left(t-\tau_{2}\right)+\gamma(t) y\left(t-\tau_{2}\right)}+\tilde{f}_{2}(t) \frac{h_{2}(t) x_{2}^{2}\left(t-\tau_{2}\right)}{e(t)+x_{2}^{2}\left(t-\tau_{2}\right)}-q(t) y(t)\right),
\end{aligned}
$$

where 


$$
\begin{aligned}
& f_{1}(t)=\frac{\omega(t) x_{1}(t)}{\omega(t) x_{1}(t)+(1-\omega(t)) x_{2}(t)}, f_{2}(t)=\frac{(1-\omega(t)) x_{2}(t)}{\omega(t) x_{1}(t)+(1-\omega(t)) x_{2}(t)} \\
& \tilde{f}_{1}(t)=\frac{\omega(t) x_{1}\left(t-\tau_{2}\right)}{\omega(t) x_{1}\left(t-\tau_{2}\right)+(1-\omega(t)) x_{2}\left(t-\tau_{2}\right)}, \tilde{f}_{2}(t)=\frac{(1-\omega(t)) x_{2}\left(t-\tau_{2}\right)}{\omega(t) x_{1}\left(t-\tau_{2}\right)+(1-\omega(t)) x_{2}\left(t-\tau_{2}\right)} .
\end{aligned}
$$

The coefficients in system (2.1) are all continuous positive $T$-periodic functions. Parameter $\omega(t)$ is the immature prey preference of the predator, which takes a value between 0 and $1 ; 1-\omega(t)$ is the mature prey preference of the predator [28] [29]. $f_{i}(t) \quad(i=1,2)$ denotes the predation weighting factor for $x_{i}(t)$. The parameters $b(t)$ and $d_{1}(t)$ represent the birth rate and the death rate of the immature prey populations, respectively. The term $b(t-\tau)_{1} \mathrm{e}^{-\int_{t-\tau_{1}}^{t} d_{1}(s) d s} x_{2}\left(t-\tau_{1}\right)$ represents the numbers of immature prey born at time $t-\tau_{1}$ that still survive, and those progressing from the immature stage to the mature stage at time $t$. The death rate of the mature prey population is logistic in nature and it is proportional to the square of the population with proportionality $d_{2}(t)$. The parameter $d_{3}(t)$ and $q(t)$ denote the death rate and the overcrowding rate or overcrowding effecting of the predator population, respectively. The overcrowding effecting $q(t)$ denote the phenomenon that population growth rate is decreased with the increase of density. The function

$c_{1}(t) x_{1}(t) /\left(\alpha(t)+\beta(t) x_{1}(t)+\gamma(t) y(t)\right)$ represents the Beddington-DeAngelis functional response of the predator to the immature prey and $c_{2}(t) / c_{1}(t)$ is the conversion rate of nutrients into the reproduction of the predator. The parameter $\tau_{2}$ is the delay due to gestation, that is to say, only the mature adult predator can contribute to the production of predator biomass. The functional response of the predator to the mature prey takes the Holling type-III form of $h_{1}(t) x_{2}^{2}(t) /\left(e(t)+x_{2}^{2}(t)\right)$ and $h_{2}(t) / h_{1}(t)$ denotes the conversion rate of nutrients into the reproduction of the predator.

The initial conditions for system (2.1) take the form of

$$
x_{i}(\theta)=\phi_{i}(\theta), y(\theta)=\psi(\theta), \phi_{i}(0)>0, \psi(0)>0, i=1,2, \theta \in[-\tau, 0],
$$

where $\tau=\max \left\{\tau_{1}, \tau_{2}\right\},\left(\phi_{1}(\theta), \phi_{2}(\theta), \psi(\theta)\right) \in C\left([-\tau, 0], R_{+0}^{3}\right)$, the Banach space of continuous functions mapping the interval $[-\tau, 0]$ into $R_{+0}^{3}$, where we define $R_{+0}^{3}=\left\{\left(x_{1}, x_{2}, x_{3}\right) \mid x_{i} \geq 0, i=1,2,3\right\}$ and the interior of $R_{+0}^{3}$ as $R_{+}^{3}=\left\{\left(x_{1}, x_{2}, x_{3}\right) \mid x_{i}>0, i=1,2,3\right\}$.

For continuity of initial conditions, we require

$$
x_{1}(0)=\int_{-\tau_{1}}^{0} b(s) \phi_{2}(s) \mathrm{e}^{-\int_{s}^{0} d_{1}(s) \mathrm{d} s} \mathrm{~d} s .
$$

For the purpose of convenience, we write

$$
B(t) \triangleq b\left(t-\tau_{1}\right) \mathrm{e}^{-\int_{t-\tau_{1}}^{t} d_{1}(s) \mathrm{d} s} .
$$

Obviously, $B(t)$ is a $T$-periodic and strictly positive function. Then system (2.1) becomes

$$
\begin{aligned}
& \dot{x}_{1}(t)=b(t) x_{2}(t)-d_{1}(t) x_{1}(t)-B(t) x_{2}\left(t-\tau_{1}\right)-f_{1}(t) \frac{c_{1}(t) x_{1}(t) y(t)}{\alpha(t)+\beta(t) x_{1}(t)+\gamma(t) y(t)}, \\
& \dot{x}_{2}(t)=B(t) x_{2}\left(t-\tau_{1}\right)-d_{2}(t) x_{2}^{2}(t)-f_{2}(t) \frac{h_{1}(t) x_{2}^{2}(t) y(t)}{e(t)+x_{2}^{2}(t)}, \\
& \dot{y}(t)=y(t)\left(-d_{3}(t)+\tilde{f}_{1}(t) \frac{c_{2}(t) x_{1}\left(t-\tau_{2}\right)}{\alpha(t)+\beta(t) x_{1}\left(t-\tau_{2}\right)+\gamma(t) y\left(t-\tau_{2}\right)}+\tilde{f}_{2}(t) \frac{h_{2}(t) x_{2}^{2}\left(t-\tau_{2}\right)}{e(t)+x_{2}^{2}\left(t-\tau_{2}\right)}-q(t) y(t)\right)
\end{aligned}
$$

In this paper, we consider system (2.5) with initial conditions (2.3) and (2.4). At the same time, we adopt the following notation through this paper:

$$
A_{T}(g)=\frac{1}{T} \int_{0}^{T} g(t) \mathrm{d} t, \quad g^{M}=\sup _{t \in[0, T]} g(t), \quad g^{l}=\inf _{t \in[0, T]} g(t),
$$

where $g(t)$ is a continuous $T$-periodic function.

The rest of the paper is arranged as follows. In the following section, we introduce some lemmas and then explore the permanence and periodicity of system (2.5). In Section 4, we investigate the extinction of the predator 
population in system (2.5). In Section 5, numerical simulations are presented to illustrate the feasibility of our main results. Furthermore, the simulated results are explained according to the biological perspective. In section 6 , a brief discussion is given to conclude this work.

\section{Permanence and Periodicity}

In this section, we analyze the permanence and periodicity of system (2.5) with initial conditions (2.3) and (2.4). Firstly, we introduce the following definition and Lemmas which are useful to obtain our result.

Definition 3.1. The system $\dot{x}(t)=F(t, x), \quad x \in R^{n}$ is said to be permanent if there are constants $M \geq m>0$ such that every positive solution of this system satisfies $m \leq \liminf _{t \rightarrow \infty} x_{i}(t) \leq \limsup _{t \rightarrow \infty} x_{i}(t) \leq M$, otherwise, the system is impermanent.

Lemma 3.2. (See [30]). If $b(t) \geq 0$ for all $t \in R$ and if $A_{T}(a(t))>0, A_{T}(b(t))>0$ then the system

$$
\dot{x}=x(a(t)-b(t) x)
$$

has a unique positive T-periodic solution which is globally asymptotically stable.

Lemma 3.3. (See [31]). System

$$
\begin{aligned}
& \dot{x}_{1}(t)=b(t) x_{2}(t)-d_{1}(t) x_{1}(t)-B(t) x_{2}\left(t-\tau_{1}\right) \\
& \dot{x}_{2}(t)=B(t) x_{2}\left(t-\tau_{1}\right)-d_{2}(t) x_{2}^{2}(t)
\end{aligned}
$$

has a unique positive T-periodic solution $\left(x_{1}^{*}(t), x_{2}^{*}(t)\right)$ which is globally asymptotically stable with respect to $R_{+0}^{2}=\left\{\left(x_{1}, x_{2}\right) \mid x_{1}>0, x_{2}>0\right\}$.

Lemma 3.4. There exists a positive constant $M_{x}$, such that

$$
\lim _{t \rightarrow+\infty} \sup x_{i}(t) \leq M_{x}, \quad(i=1,2)
$$

for all the solution of system (3.2) with respect to $R_{+0}^{2}=\left\{\left(x_{1}, x_{2}\right) \mid x_{1}>0, x_{2}>0\right\}$.

Proof. Let $\left(x_{1}(t), x_{2}(t)\right) \in R_{+0}^{2}$ be the any solution of system (3.2). By Lemma 3.3, system (3.2) has a unique globally attractive positive $T$-periodic solution $\left(x_{1}^{*}(t), x_{2}^{*}(t)\right)$. From the global attractivity of $\left(x_{1}^{*}(t), x_{2}^{*}(t)\right)$, for any positive constant $\varepsilon \quad(0<\varepsilon<1)$, there exists a $T_{1}>0$, such that for all $t \geq T_{1}$

$$
\left|x_{i}(t)-x_{i}^{*}(t)\right|<\varepsilon, i=1,2 \text {. }
$$

By applying (3.4), we obtain $x_{i}(t) \leq x_{i}^{*}(t)+\varepsilon, \quad i=1,2, \quad t \geq T_{1}$.

Let

$$
M_{x}=\max _{0 \leq t \leq T}\left\{x_{i}^{*}(t)+\varepsilon, i=1,2\right\} .
$$

We have $\lim _{t \rightarrow+\infty} \sup x_{i}(t) \leq M_{x}, \quad(i=1,2)$.

Theorem 3.5. System (2.5) is permanent and has at least one positive T-periodic solution provided

$$
A_{T}\left(-d_{3}(t)+\tilde{f}_{1}^{*}(t) \frac{c_{2}(t) x_{1}^{*}\left(t-\tau_{2}\right)}{\alpha(t)+\beta(t) x_{1}^{*}\left(t-\tau_{2}\right)}+\tilde{f}_{2}^{*}(t) \frac{h_{2}(t)\left(x_{2}^{*}\left(t-\tau_{2}\right)\right)^{2}}{e(t)+\left(x_{2}^{*}\left(t-\tau_{2}\right)\right)^{2}}\right)>0,
$$

where

$$
\tilde{f}_{1}^{*}(t)=\frac{\omega(t) x_{1}^{*}\left(t-\tau_{2}\right)}{\omega(t) x_{1}^{*}\left(t-\tau_{2}\right)+(1-\omega(t)) M_{x}}, \quad \tilde{f}_{2}^{*}(t)=\frac{(1-\omega(t)) x_{2}^{*}\left(t-\tau_{2}\right)}{\omega(t) M_{x}+(1-\omega(t)) x_{2}^{*}\left(t-\tau_{2}\right)},
$$

where $\left(x_{1}^{*}(t), x_{2}^{*}(t)\right)$ is the unique positive periodic solution of system (3.2) given by Lemma 3.3 and $M_{x}$ is the upper bound of system (3.2) given by Lemma 3.4 and defined by equation (3.5).

We need the following propositions to prove Theorem 3.5.

Proposition 3.6. For all the solutions of system (2.5) with initial conditions (2.3) and (2.4), we have $\lim _{t \rightarrow+\infty} \sup x_{i}(t) \leq M_{x},(i=1,2)$, where $M_{x}$ is the upper bound of system (3.2) given by Lemma 3.4 and defined by equation (3.5). Furthermore, there exists a positive constant $M_{y}$, such that $\lim _{t \rightarrow+\infty} \sup y(t) \leq M_{y}$.

Proof. Obviously, $R_{+}^{3}=\left\{\left(x_{1}(t), x_{2}(t), y(t)\right) \mid x_{i}(t) \geq 0, y(t) \geq 0\right\}$ is a positively invariant set of system (2.5). 
Given any solution $\left(x_{1}(t), x_{2}(t), y(t)\right)$ of system (2.5) with initial conditions (2.3) and (2.4), we have

$$
\begin{gathered}
\dot{x}_{1}(t) \leq b(t) x_{2}(t)-d_{1}(t) x_{1}(t)-B(t) x_{2}\left(t-\tau_{1}\right), \\
\dot{x}_{2}(t) \leq B(t) x_{2}\left(t-\tau_{1}\right)-d_{2}(t) x_{2}^{2}(t) .
\end{gathered}
$$

Consider the following auxiliary system

$$
\begin{aligned}
& \dot{u}_{1}(t)=b(t) u_{2}(t)-d_{1}(t) u_{1}(t)-B(t) u_{2}\left(t-\tau_{1}\right), \\
& \dot{u}_{2}(t)=B(t) u_{2}\left(t-\tau_{1}\right)-d_{2}(t) u_{2}^{2}(t) .
\end{aligned}
$$

By Lemma 3.3, system (3.7) has a unique globally attractive positive $T$-periodic solution $\left(x_{1}^{*}(t), x_{2}^{*}(t)\right)$. Let $\left(u_{1}(t), u_{2}(t)\right)$ be the solution of system (3.7) with $\left(u_{1}(0), u_{2}(0)\right)=\left(x_{1}(0), x_{2}(0)\right)$. By the vector comparison theorem [32], we have

$$
x_{i}(t) \leq u_{i}(t),(i=1,2), t \geq 0 .
$$

By applying (3.8) and Lemma 3.4, we obtain $\lim _{t \rightarrow+\infty} \sup x_{i}(t) \leq \lim _{t \rightarrow+\infty} \sup u_{i}(t) \leq M_{x}, \quad i=1,2$.

In addition, from the third equation of (2.5) we have

$$
\dot{y}(t) \leq y(t)\left(-d_{3}(t)+\frac{c_{2}(t)}{\beta(t)}+h_{2}(t)-q(t) y(t)\right) .
$$

Consider the following auxiliary equation:

$$
\dot{v}(t)=v(t)\left(-d_{3}(t)+\frac{c_{2}(t)}{\beta(t)}+h_{2}(t)-q(t) v(t)\right) .
$$

According to the condition (3.6), we have

$$
A_{T}\left(-d_{3}(t)+\frac{c_{2}(t)}{\beta(t)}+h_{2}(t)\right)>0 .
$$

By (3.10) and Lemma (3.2), we obtain that system (3.9) has a unique positive $T$-periodic solution $y^{*}(t)>0$ which is globally asymptotically stable. Then, for the above $\varepsilon$ given in (3.4), there exists a $T_{2}>T_{1}$, such that

$$
\left|y(t)-y^{*}(t)\right|<\varepsilon, t \geq T_{2},
$$

By applying (3.11), we obtain $y(t)<y^{*}(t)+\varepsilon, t \geq T_{2}$.

Set $M_{y}=\max _{0 \leq t \leq T}\left\{y^{*}(t)+\varepsilon\right\}$, then

$$
\lim _{t \rightarrow+\infty} \sup y(t) \leq M_{y} .
$$

This completes the proof of Proposition 3.6.

Proposition 3.7. There exists a positive constant $\eta_{x}<M_{x}$, such that $\lim _{t \rightarrow+\infty} \inf x_{i}(t) \geq \eta_{x}, \quad i=1,2$.

Proof. By Proposition 3.6, there exists a positive $T_{2}>0$ such that $0<y(t)<M_{y}, t \geq T_{2}$. Hence, from the first and second equations of system (2.5), we obtain

$$
\begin{gathered}
\dot{x}_{1}(t) \geq b(t) x_{2}(t)-\left(d_{1}(t)+f_{1}(t) \frac{c_{1}(t) M_{y}}{\alpha(t)}\right) x_{1}(t)-B(t) x_{2}\left(t-\tau_{1}\right), \\
\dot{x}_{2}(t) \geq B(t) x_{2}\left(t-\tau_{1}\right)-\left(d_{2}(t)+f_{2}(t) \frac{h_{1}(t) M_{y}}{e(t)}\right) x_{2}^{2}(t),
\end{gathered}
$$

for $t \geq T_{2}$. By Lemma 3.3, the following auxiliary system

$$
\begin{aligned}
& \dot{u}_{1}(t)=b(t) u_{2}(t)-\left(d_{1}(t)+f_{1}(t) \frac{c_{1}(t) M_{y}}{\alpha(t)}\right) u_{1}(t)-B(t) u_{2}\left(t-\tau_{1}\right), \\
& \dot{u}_{2}(t)=B(t) u_{2}\left(t-\tau_{1}\right)-\left(d_{2}(t)+f_{2}(t) \frac{h_{1}(t) M_{y}}{e(t)}\right) u_{2}^{2}(t),
\end{aligned}
$$


has a unique global attractive positive $T$-periodic solution $\left(\tilde{x}_{1}^{*}(t), \tilde{x}_{2}^{*}(t)\right)$. Let $\left(u_{1}(t), u_{2}(t)\right)$ be the solution of system (3.13) with $\left(u_{1}\left(T_{2}\right), u_{2}\left(T_{2}\right)\right)=\left(x_{1}\left(T_{2}\right), x_{2}\left(T_{2}\right)\right)$, by the vector comparison theorem [32], we obtain

$$
x_{i}(t) \geq u_{i}(t),(i=1,2), t \geq T_{2} \text {. }
$$

Moreover, from the global attractivity of $\left(\tilde{x}_{1}^{*}(t), \tilde{x}_{2}^{*}(t)\right)$, there exists a $T_{3}>T_{2}$, such that

$$
\left|u_{i}(t)-\tilde{x}_{i}^{*}(t)\right|<\frac{\tilde{x}_{i}^{*}(t)}{2}, t \geq T_{3} .
$$

Combined (3.14) with (3.15), we have

$$
x_{i}(t)>\min \left\{\min _{t \in[0, T]}\left\{\frac{\tilde{x}_{i}^{*}(t)}{2}, i=1,2\right\}\right\} \triangleq \eta_{x}, t \geq T_{3}
$$

Therefore, $\lim _{t \rightarrow+\infty} \inf x_{i}(t) \geq \eta_{x}, \quad i=1,2$. This completes the proof of Proposition 3.7.

Proposition 3.8. Suppose that (3.6) holds, then there exists a positive constant $\eta_{y}$, such that any solution $\left(x_{1}(t), x_{2}(t), y(t)\right)$ of system (2.5) with initial conditions (2.3) and (2.4) satisfies

$$
\lim _{t \rightarrow+\infty} \sup y(t) \geq \eta_{y} \text {. }
$$

Proof. By assumption (3.6), we can choose arbitrarily small constant $\varepsilon_{0}>0$ (without loss generality, we assume that $0<\varepsilon_{0}<\frac{1}{2} \min _{t \in[0, T]}\left\{x_{1}^{*}(t), x_{2}^{*}(t)\right\}$, where $\left(x_{1}^{*}(t), x_{2}^{*}(t)\right)$ is the unique positive periodic solution of system (3.2)), such that

$$
A_{T}\left(\phi_{\varepsilon_{0}}(t)\right)>0
$$

where

$$
\begin{aligned}
& \phi_{\varepsilon_{0}}(t)=-d_{3}(t)+\tilde{f}_{1}^{*}\left(t, \varepsilon_{0}\right) \frac{c_{2}(t)\left(x_{1}^{*}\left(t-\tau_{2}\right)-\varepsilon_{0}\right)}{\alpha(t)+\beta(t)\left(x_{1}^{*}\left(t-\tau_{2}\right)-\varepsilon_{0}\right)+\gamma(t) \varepsilon_{0}}+\tilde{f}_{2}^{*}\left(t, \varepsilon_{0}\right) \frac{h_{2}(t)\left(x_{2}^{*}\left(t-\tau_{2}\right)-\varepsilon_{0}\right)^{2}}{e(t)+\left(x_{2}^{*}\left(t-\tau_{2}\right)-\varepsilon_{0}\right)^{2}}-q(t) \varepsilon_{0}, \\
& \tilde{f}_{1}^{*}\left(t, \varepsilon_{0}\right)=\frac{\omega(t)\left(x_{1}^{*}\left(t-\tau_{2}\right)-\varepsilon_{0}\right)}{\omega(t)\left(x_{1}^{*}\left(t-\tau_{2}\right)-\varepsilon_{0}\right)+(1-\omega(t)) M_{x}}, \quad \tilde{f}_{2}^{*}\left(t, \varepsilon_{0}\right)=\frac{(1-\omega(t))\left(x_{2}^{*}\left(t-\tau_{2}\right)-\varepsilon_{0}\right)}{\omega(t) M_{x}+(1-\omega(t))\left(x_{2}^{*}\left(t-\tau_{2}\right)-\varepsilon_{0}\right)} .
\end{aligned}
$$

Consider the following system with a parameter $\delta$,

$$
\begin{gathered}
x_{1}^{\prime}(t)=b(t) x_{2}(t)-\left(d_{1}(t)+2 \delta f_{1}(t) \frac{c_{1}(t)}{\alpha(t)}\right) x_{1}(t)-B(t) x_{2}\left(t-\tau_{1}\right), \\
x_{2}^{\prime}(t)=B(t) x_{2}\left(t-\tau_{1}\right)-\left(d_{2}(t)+2 \delta f_{2}(t) \frac{h_{1}(t)}{e(t)}\right) x_{2}^{2}(t),
\end{gathered}
$$

By Lemma 3.3, system (3.19) has a unique positive $T$-periodic solution $\left(x_{1 \delta}^{*}(t), x_{2 \delta}^{*}(t)\right)$, which is globally attractive. Let $\left(\bar{x}_{1 \delta}(t), \bar{x}_{2 \delta}(t)\right)$ be the solution (3.19) with initial condition $\left(\bar{x}_{1 \delta}(0), \bar{x}_{2 \delta}(0)\right)=\left(x_{1}^{*}(0), x_{2}^{*}(0)\right)$. Then, for the above $\varepsilon_{0}$, there exists a sufficiently large $T_{4}>T_{3}$ such that $\left|\bar{x}_{i \delta}(t)-x_{i \delta}^{*}(t)\right|<\frac{\varepsilon_{0}}{4}$, for $i=1,2$, $t>T_{4}$.

Using the continuity of the solution in the parameter, we have $\bar{x}_{i \delta}(t) \rightarrow x_{i}^{*}(t)$ uniformly in $\left[T_{4}, T_{4}+T\right]$ as $\delta \rightarrow 0$. Hence, there exists a $\delta_{0}=\delta_{0}\left(\varepsilon_{0}\right)>0$ such that $\left|\bar{x}_{i \delta}(t)-x_{i}^{*}(t)\right|<\frac{\varepsilon_{0}}{4}$, for $i=1,2, t \in\left[T_{4}, T_{4}+T\right]$, $0<\delta<\delta_{0}$.

So, we get $\left|x_{i \delta}^{*}(t)-x_{i}^{*}(t)\right| \leq\left|\bar{x}_{i \delta}(t)-x_{i \delta}^{*}(t)\right|+\left|\bar{x}_{i \delta}(t)-x_{i}^{*}(t)\right|<\frac{\varepsilon_{0}}{2}$. Choosing a constant $\delta_{1} \quad\left(0<\delta_{1}<\delta_{0}\right.$, $2 \delta_{1}<\varepsilon_{0}$ ), we obtain

$$
x_{i \delta_{1}}^{*}(t) \geq x_{i}^{*}(t)-\frac{\varepsilon_{0}}{2}, \quad i=1,2, \quad t \geq 0
$$


Suppose that the conclusion (3.17) is not true, then there exists $\psi \in C_{+}$such that

$$
\lim _{t \rightarrow+\infty} \sup y(t)<\delta_{1} \text {, }
$$

where $\left(x_{1}(t, \psi), x_{2}(t, \psi), y(t, \psi)\right)$ is the solution of system (2.5) with initial condition $\left(x_{1}(\theta), x_{2}(\theta), y(\theta)\right)=\psi(\theta), \theta \in[-\tau, 0]$. So, there exists $T_{5}>T_{4}$ such that

$$
y(t)<2 \delta_{1}<\varepsilon_{0}, \quad t \geq T_{5} .
$$

By applying (3.21), from the first and second equation of system (2.5), we have

$$
\begin{gathered}
\dot{x}_{1}(t, \psi) \geq b(t) x_{2}(t, \psi)-\left(d_{1}(t)+2 \delta_{1} f_{1}(t) \frac{c_{1}(t)}{\alpha(t)}\right) x_{1}(t, \psi)-B(t) x_{2}\left(t-\tau_{1}, \psi\right), \\
\dot{x}_{2}(t, \psi) \geq B(t) x_{2}\left(t-\tau_{1}, \psi\right)-\left(d_{2}(t)+2 \delta_{1} f_{2}(t) \frac{h_{1}(t)}{e(t)}\right) x_{2}^{2}(t, \psi),
\end{gathered}
$$

for all $t \geq T_{6} \geq T_{5}+\tau_{1}$. Let $\left(u_{1}(t), u_{2}(t)\right)$ be the solution of system (3.19) with $\delta=\delta_{1}$ and $\left(u_{1}\left(T_{6}\right), u_{2}\left(T_{6}\right)\right)=\left(x_{1}\left(T_{6}, \psi\right), x_{2}\left(T_{6}, \psi\right)\right)$, then we have $x_{i}(t, \psi) \geq u_{i}(t), i=1,2, t \geq T_{6}$. By the global asymptotic stability of $\left(x_{1 \delta_{1}}^{*}(t), x_{2 \delta_{1}}^{*}(t)\right)$, for the given $\varepsilon=\varepsilon_{0} / 2$, there exists $T_{7} \geq T_{6}$, such that

$$
\left|u_{i}(t)-x_{i \delta_{1}}^{*}(t)\right|<\frac{\varepsilon_{0}}{2}, \quad i=1,2, t \geq T_{7} .
$$

So,

$$
x_{i}(t, \psi) \geq u_{i}(t)>x_{i \delta_{1}}^{*}(t)-\frac{\varepsilon_{0}}{2}, i=1,2, \quad t \geq T_{7} .
$$

By using (3.20), we obtain

$$
x_{i}(t, \psi) \geq x_{i}^{*}(t)-\varepsilon_{0}, \quad i=1,2, \quad t \geq T_{7} .
$$

Therefore, by using (3.21) and (3.22), for $t \geq T_{7}+\tau_{2}$ it follows

$$
\begin{aligned}
\dot{y}(t, \psi) & \geq y(t, \psi)\left(-d_{3}(t)+\tilde{f}_{1}^{*}(t) \frac{c_{2}(t)\left(x_{1}^{*}\left(t-\tau_{2}\right)-\varepsilon_{0}\right)}{\alpha(t)+\beta(t)\left(x_{1}^{*}\left(t-\tau_{2}\right)-\varepsilon_{0}\right)+\gamma(t) \varepsilon_{0}}+\tilde{f}_{2}^{*}(t) \frac{h_{2}(t)\left(x_{2}^{*}\left(t-\tau_{2}\right)-\varepsilon_{0}\right)^{2}}{e(t)+\left(x_{2}^{*}\left(t-\tau_{2}\right)-\varepsilon_{0}\right)^{2}}-q(t) \varepsilon_{0}\right) \\
& =y(t, \psi) \phi_{\varepsilon_{0}}(t) .
\end{aligned}
$$

Integrating (3.23) from $T_{7}+\tau_{2}$ to $t$ yields

$$
y(t, \psi) \geq y\left(T_{7}+\tau_{2}, \psi\right) \exp \int_{T_{7}+\tau_{2}}^{t} \phi_{\varepsilon_{0}}(t) \mathrm{d} t
$$

Thus, from (3.18) we know that $y(t, \psi) \rightarrow+\infty$ as $t \rightarrow+\infty$, which is a contradiction. The proof is complete.

Proposition 3.9. Suppose that (3.6) holds, then there exists a positive constant $\rho_{y}$, such that any solution $\left(x_{1}(t), x_{2}(t), y(t)\right)$ of system (2.5) with initial conditions (2.3) and (2.4) satisfies

$$
\lim _{t \rightarrow+\infty} \inf y(t) \geq \rho_{y} .
$$

Proof. Suppose that (3.24) is not true, then there exists a sequence $\left\{\xi_{m}\right\} \in C_{+}$, such that

$$
\lim _{t \rightarrow+\infty} \inf \left(y, \xi_{m}\right)<\frac{\eta_{y}}{(m+1)^{2}}, \quad m=1,2, \cdots
$$

On the other hand, by Proposition 3.8, we have

$$
\lim _{t \rightarrow+\infty} \sup y\left(t, \xi_{m}\right) \geq \eta_{y}, \quad m=1,2, \cdots
$$


Hence, there exist time sequences $\left\{s_{q}^{(m)}\right\}$ and $\left\{t_{q}^{(m)}\right\}$ satisfying

$$
0<s_{1}^{(m)}<t_{1}^{(m)}<s_{2}^{(m)}<t_{2}^{(m)}<\cdots<s_{q}^{(m)}<t_{q}^{(m)}<\cdots, \quad s_{q}^{(m)} \rightarrow+\infty, t_{q}^{(m)} \rightarrow+\infty \text { as } q \rightarrow+\infty,
$$

and

$$
y\left(s_{q}^{(m)}, \xi_{m}\right)=\frac{\eta_{y}}{m+1}, \quad y\left(t_{q}^{(m)}, \xi_{m}\right)=\frac{\eta_{y}}{(m+1)^{2}}, \frac{\eta_{y}}{(m+1)^{2}}<y\left(t, \xi_{m}\right)<\frac{\eta_{y}}{m+1}, \quad t \in\left(s_{q}^{(m)}, t_{q}^{(m)}\right) .
$$

By Proposition 3.6, for a given positive integer $m$, there exist a $T_{1}^{(m)}>0$, such that for all $t>T_{1}^{(m)}$

$$
y\left(t, \xi_{m}\right)<M_{y}, \quad m=1,2, \cdots .
$$
hence

Because of $s_{q}^{(m)} \rightarrow+\infty$ as $q \rightarrow+\infty$, there is a positive integer $K^{(m)}$, such that $s_{q}^{(m)}>T_{1}^{(m)}$ as $q \geq K^{(m)}$,

$$
\dot{y}\left(t, \xi_{m}\right) \geq y\left(t, \xi_{m}\right)\left(-d_{3}(t)-q(t) M_{y}\right),
$$

for $t \in\left[s_{q}^{(m)}, t_{q}^{(m)}\right], \quad q \geq K^{(m)}$. Integrating (3.26) from $s_{q}^{(m)}$ to $t_{q}^{(m)}$ yields

$$
y\left(t_{q}^{(m)}, \xi_{m}\right) \geq y\left(s_{q}^{(m)}, \xi_{m}\right) \exp \left(\int_{s_{q}^{(m)}}^{t_{q}^{(m)}}\left(-d_{3}(t)-q(t) M_{y}\right) \mathrm{d} t\right),
$$

or

$$
\int_{s_{q}^{(m)}}^{t_{q}^{(m)}}\left(d_{3}(t)+q(t) M_{y}\right) \mathrm{d} t \geq \ln (m+1), \quad q \geq K^{(m)} .
$$

Thus, from the boundedness of $d_{3}(t)+q(t) M_{y}$, we have

$$
t_{q}^{(m)}-s_{q}^{(m)} \rightarrow+\infty \text { as } m \rightarrow+\infty, q \geq K^{(m)} .
$$

By (3.18) and (3.27), there exist constants $P>0$ and $N_{0}>0$, such that

$$
\frac{\eta_{y}}{m+1}<\delta_{1}<\varepsilon_{0}, \quad t_{q}^{(m)}-s_{q}^{(m)}>2 P,
$$

and $\int_{0}^{a} \phi_{\varepsilon_{0}}(t) \mathrm{d} t>0$ for $m \geq N_{0}, \quad q \geq K^{(m)}$ and $a \geq P$. (3.28) implies that

$$
y\left(t, \xi_{m}\right)<\delta_{1}<\varepsilon_{0}, \quad t \in\left[s_{q}^{(m)}, t_{q}^{(m)}\right],
$$

for $m \geq N_{0}, q \geq K^{(m)}$. In addition, for $t \in\left[s_{q}^{(m)}, t_{q}^{(m)}\right]$, we have

$$
\begin{gathered}
\dot{x}_{1}\left(t, \xi_{m}\right) \geq b(t) x_{2}\left(t, \xi_{m}\right)-\left(d_{1}(t)+2 \delta_{1} f_{1}(t) \frac{c_{1}(t)}{\alpha(t)}\right) x_{1}\left(t, \xi_{m}\right)-B(t) x_{2}\left(t-\tau_{1}, \xi_{m}\right), \\
\dot{x}_{2}\left(t, \xi_{m}\right) \geq B(t) x_{2}\left(t-\tau_{1}, \xi_{m}\right)-\left(d_{2}(t)+2 \delta_{1} f_{2}(t) \frac{h_{1}(t)}{e(t)}\right) x_{2}^{2}\left(t, \xi_{m}\right) .
\end{gathered}
$$

Let $\left(u_{1}(t), u_{2}(t)\right)$ be the solution of (3.19) with $\delta=\delta_{1}$ and $u_{i}\left(s_{q}^{(m)}\right)=x_{i}\left(s_{q}^{(m)}, \xi_{m}\right)$, then by applying comparison theorem, we have

$$
x_{i}\left(t, \xi_{m}\right) \geq u_{i}(t), \quad t \in\left[s_{q}^{(m)}, t_{q}^{(m)}\right] .
$$

By using Propositions 3.6 and 3.7, there exists a large enough $K_{1}^{(m)}>K^{(m)}$ such that

$$
\eta_{x}<x_{i}\left(s_{q}^{(m)}, \xi_{m}\right)<M_{x},
$$

for $q \geq K_{1}^{(m)}$. For $\delta=\delta_{1}$, system (3.19) has a unique positive $T$-periodic solution $\left(x_{1 \delta_{1}}^{*}(t), x_{2 \delta_{1}}^{*}(t)\right)$ which is global stability. According to the periodicity of (3.19), we have the periodic solution $\left(x_{1 \delta_{1}}^{*}(t), x_{2 \delta_{1}}^{*}(t)\right)$ is un- 
iformly asymptotically stable in the compact set $\Omega=\left\{x \mid \eta_{x}<x<M_{x}\right\}$. Hence, for the given $\varepsilon_{0}$ in Proposition 3.8, there exists $T_{0}=T_{0}\left(\varepsilon_{0}\right)>P+\tau_{2}$, which is independent of $m$ and $q$, such that

$$
u_{1}(t)>x_{1 \delta_{1}}^{*}(t)-\frac{\varepsilon_{0}}{2}, t>T_{0}+s_{q}^{(m)} .
$$

Therefore, by using (3.20),

$$
u_{1}(t)>x_{1}^{*}(t)-\varepsilon_{0}, \quad t>T_{0}+s_{q}^{(m)} .
$$

According to (3.27), there exists a positive integer $N_{1} \geq N_{0}$ such that $t_{q}^{(m)} \geq s_{q}^{(m)}+2 T_{0}>s_{q}^{(m)}+2\left(P+\tau_{2}\right)$ for $m \geq N_{1}$ and $q \geq K_{1}^{(m)}$. Thus, we obtain

$$
x_{1}\left(t, \xi_{m}\right) \geq x_{1}^{*}(t)-\varepsilon_{0}, \quad t \in\left[T_{0}+s_{q}^{(m)}, t_{q}^{(m)}\right],
$$

as $m \geq N_{1}$ and $q \geq K^{(m)}$. Therefore, by using (3.29) and (3.30), from the third equation of system (2.5), we have

$$
\dot{y}\left(t, \xi_{m}\right) \geq y\left(t, \xi_{m}\right) \phi_{\varepsilon_{0}}(t), \quad t \in\left[T_{0}+s_{q}^{(m)}, t_{q}^{(m)}\right] .
$$

Integrating (3.31) from $T_{0}+s_{q}^{(m)}+\tau_{2}$ to $t_{q}^{(m)}$ leads to

$$
y\left(t_{q}^{(m)}, \xi_{m}\right) \geq y\left(T_{0}+s_{q}^{(m)}+\tau_{2}, \xi_{m}\right) \exp \left(\int_{T_{0}+s_{q}^{(m)}+\tau_{2}}^{t_{q}^{(m)}} \phi_{\varepsilon_{0}}(t) \mathrm{d} t\right),
$$

that is,

$$
\frac{\eta_{y}}{(m+1)^{2}} \geq \frac{\eta_{y}}{(m+1)^{2}} \exp \left(\int_{T_{0}+s_{q}^{(m)}+\tau_{2}}^{t_{(m)}^{(m)}} \phi_{\varepsilon_{0}}(t) \mathrm{d} t\right)>\frac{\eta_{y}}{(m+1)^{2}} .
$$

This is a contradiction. This completes the proof of Proposition 3.9.

Proof of Theorem 3.5. By using Propositions 3.6-3.9, system (2.5) is permanent. Using result given by Teng and Chen in [33], we obtain system (2.5) has at least one positive $T$-periodic solution. This completes the proof of Theorem 3.5.

\section{Extinction}

In this section, we investigate the extinction of the predator population in system (2.5) with initial conditions (2.3) and (2.4) under some condition.

Theorem 4.1. Suppose that

$$
A_{T}\left(-d_{3}(t)+\bar{f}_{1}^{*}(t) \frac{c_{2}(t) x_{1}^{*}\left(t-\tau_{2}\right)}{\alpha(t)+\beta(t) x_{1}^{*}\left(t-\tau_{2}\right)}+\bar{f}_{2}^{*}(t) \frac{h_{2}(t)\left(x_{2}^{*}\left(t-\tau_{2}\right)\right)^{2}}{e(t)+\left(x_{2}^{*}\left(t-\tau_{2}\right)\right)^{2}}\right) \leq 0 .
$$

where

$$
\bar{f}_{1}^{*}(t)=\frac{\omega(t) x_{1}^{*}\left(t-\tau_{2}\right)}{\omega(t) x_{1}^{*}\left(t-\tau_{2}\right)+(1-\omega(t)) \eta_{x}}, \quad \bar{f}_{2}^{*}(t)=\frac{(1-\omega(t)) x_{2}^{*}\left(t-\tau_{2}\right)}{\omega(t) \eta_{x}+(1-\omega(t)) x_{2}^{*}\left(t-\tau_{2}\right)},
$$

where $\left(x_{1}^{*}(t), x_{2}^{*}(t)\right)$ is the unique positive periodic solution of system (3.2) given by Lemma 3.3 and $\eta_{x}$ is the lower bound of system (3.2) given by Proposition 3.7 and defined by equation (3.16), then $\lim y(t)=0$.

Proof. According to (4.1), for every given positive constant $\varepsilon \quad(0<\varepsilon<1)$, there exists $\varepsilon_{1}^{t \rightarrow+\infty}>0 \quad\left(0<\varepsilon_{1}<\varepsilon\right)$ and $\varepsilon_{0}>0$ such that

$$
\begin{aligned}
& A_{T}\left(-d_{3}(t)+\bar{f}_{1}^{*}\left(t, \varepsilon_{1}\right) \frac{c_{2}(t)\left(x_{1}^{*}\left(t-\tau_{2}\right)+\varepsilon_{1}\right)}{\alpha(t)+\beta(t)\left(x_{1}^{*}\left(t-\tau_{2}\right)+\varepsilon_{1}\right)}+\bar{f}_{2}^{*}\left(t, \varepsilon_{1}\right) \frac{h_{2}(t)\left(x_{2}^{*}\left(t-\tau_{2}\right)+\varepsilon_{1}\right)^{2}}{e(t)+\left(x_{2}^{*}\left(t-\tau_{2}\right)+\varepsilon_{1}\right)^{2}}-q(t) \varepsilon\right) \\
& \leq-\frac{\varepsilon}{2} A_{T}(q(t))<-\varepsilon_{0},
\end{aligned}
$$

where 


$$
\bar{f}_{1}^{*}\left(t, \varepsilon_{1}\right)=\frac{\omega(t)\left(x_{1}^{*}\left(t-\tau_{2}\right)+\varepsilon_{1}\right)}{\omega(t)\left(x_{1}^{*}\left(t-\tau_{2}\right)+\varepsilon_{1}\right)+(1-\omega(t)) \eta_{x}}, \quad \bar{f}_{2}^{*}\left(t, \varepsilon_{1}\right)=\frac{(1-\omega(t))\left(x_{2}^{*}\left(t-\tau_{2}\right)+\varepsilon_{1}\right)}{\omega(t) \eta_{x}+(1-\omega(t))\left(x_{2}^{*}\left(t-\tau_{2}\right)+\varepsilon_{1}\right)} .
$$

From the first and second equations of system (2.5), we have

$$
\begin{gathered}
\dot{x}_{1}(t) \leq b(t) x_{2}(t)-d_{1}(t) x_{1}(t)-B(t) x_{2}\left(t-\tau_{1}\right), \\
\dot{x}_{2}(t) \leq B(t) x_{2}\left(t-\tau_{1}\right)-d_{2}(t) x_{2}^{2}(t) .
\end{gathered}
$$

Hence, for the above $\varepsilon_{1}$ there are exists a $\Gamma^{(1)}>0$, such that

$$
x_{i}(t)<x_{i}^{*}(t)+\varepsilon_{1}, \quad i=1,2, \quad t \geq \Gamma^{(1)} .
$$

It follows from (4.2) and (4.3) that for $t \geq \Gamma^{(1)}+\tau_{2}$,

$$
A_{T}\left(-d_{3}(t)+\bar{f}_{1}(t) \frac{c_{2}(t) x_{1}\left(t-\tau_{2}\right)}{\alpha(t)+\beta(t) x_{1}\left(t-\tau_{2}\right)}+\bar{f}_{2}(t) \frac{h_{2}(t) x_{2}^{2}\left(t-\tau_{2}\right)}{e(t)+x_{2}^{2}\left(t-\tau_{2}\right)}-q(t) \varepsilon\right) \leq-\varepsilon_{0} .
$$

Firstly, we show that exists a $\Gamma^{(2)}>\Gamma^{(1)}$, such that $y\left(\Gamma^{(2)}\right)<\varepsilon$. Otherwise, by (4.4), we have

$$
\begin{aligned}
& \varepsilon \leq y(t) \\
& \leq y\left(\Gamma^{(1)}+\tau_{2}\right) \exp \left\{\left(\int_{\Gamma^{(1)}+\tau_{2}}^{t}-d_{3}(s)+\bar{f}_{1}(s) \frac{c_{2}(s) x_{1}\left(s-\tau_{2}\right)}{\alpha(s)+\beta(s) x_{1}\left(s-\tau_{2}\right)}+\bar{f}_{2}(s) \frac{h_{2}(s) x_{2}^{2}\left(s-\tau_{2}\right)}{e(s)+x_{2}^{2}\left(s-\tau_{2}\right)}-q(s) \varepsilon\right) \mathrm{d} s\right\} \\
& \leq y\left(\Gamma^{(1)}+\tau_{2}\right) \exp \left\{-\varepsilon_{0}\left(t-\left(\Gamma^{(1)}+\tau_{2}\right)\right)\right\} \rightarrow 0, \text { as } t \rightarrow+\infty .
\end{aligned}
$$

That is to say $\varepsilon \leq 0$. This is a contradiction.

Secondly, we show that

$$
y(t) \leq \varepsilon \exp \{M(\varepsilon) T\}, \text { for } t \geq \Gamma^{(2)},
$$

where

$$
M(\varepsilon)=\max _{0 \leq t \leq T}\left(d_{3}(t)+\bar{f}_{1}(t) \frac{c_{2}(t) x_{1}\left(t-\tau_{2}\right)}{\alpha(t)+\beta(t) x_{1}\left(t-\tau_{2}\right)}+\bar{f}_{2}(t) \frac{h_{2}(t) x_{2}^{2}\left(t-\tau_{2}\right)}{e(t)+x_{2}^{2}\left(t-\tau_{2}\right)}+q(t) \varepsilon\right),
$$

is bounded for $\varepsilon \in[0,1]$. Otherwise, there exists a $\Gamma^{(3)}>\Gamma^{(2)}$ such that $y\left(\Gamma^{(3)}\right)>\varepsilon \exp \{M(\varepsilon) T\}$.

By the continuity of $y(t)$, there must exists a $\Gamma^{(4)} \in\left(\Gamma^{(2)}, \Gamma^{(3)}\right)$ such that $y\left(\Gamma^{(4)}\right)=\varepsilon$ and $y(t)>\varepsilon$ for $t \in\left(\Gamma^{(4)}, \Gamma^{(3)}\right]$. Let $P_{1}$ be the nonnegative integer such that $\Gamma^{(3)} \in\left(\Gamma^{(4)}+P_{1} T, \Gamma^{(4)}+\left(P_{1}+1\right) T\right)$. According to (4.3), we have

$$
\begin{aligned}
& \varepsilon \exp \{M(\varepsilon) T\}<y\left(\Gamma^{(3)}\right) \\
& <y\left(\Gamma^{(4)}\right) \exp \left\{\int_{\Gamma^{(4)}}^{\Gamma^{(3)}}\left(-d_{3}(t)+\bar{f}_{1}(t) \frac{c_{2}(t) x_{1}\left(t-\tau_{2}\right)}{\alpha(t)+\beta(t) x_{1}\left(t-\tau_{2}\right)}+\bar{f}_{2}(t) \frac{h_{2}(t) x_{2}^{2}\left(t-\tau_{2}\right)}{e(t)+x_{2}^{2}\left(t-\tau_{2}\right)}-q(t) \varepsilon\right) \mathrm{d} t\right\} \\
& =\varepsilon \exp \left\{\int_{\Gamma^{(4)}}^{\Gamma^{(4)}+R_{1} T}+\int_{\Gamma^{(4)}+R_{1} T}^{\Gamma^{(3)}}\right\}\left(-d_{3}(t)+\bar{f}_{1}(t) \frac{c_{2}(t) x_{1}\left(t-\tau_{2}\right)}{\alpha(t)+\beta(t) x_{1}\left(t-\tau_{2}\right)}+\bar{f}_{2}(t) \frac{h_{2}(t) x_{2}^{2}\left(t-\tau_{2}\right)}{e(t)+x_{2}^{2}\left(t-\tau_{2}\right)}-q(t) \varepsilon\right) \mathrm{d} t \\
& <\varepsilon \exp \left\{\int_{\Gamma^{(4)}+P_{1} T}^{\Gamma^{(3)}}\left(d_{3}(t)+\bar{f}_{1}(t) \frac{c_{2}(t) x_{1}\left(t-\tau_{2}\right)}{\alpha(t)+\beta(t) x_{1}\left(t-\tau_{2}\right)}+\bar{f}_{2}(t) \frac{h_{2}(t) x_{2}^{2}\left(t-\tau_{2}\right)}{e(t)+x_{2}^{2}\left(t-\tau_{2}\right)}+q(t) \varepsilon\right) \mathrm{d} t\right\} \\
& \leq \varepsilon \exp \{M(\varepsilon) T\},
\end{aligned}
$$

which is a contradiction. This shows that (4.5) holds. By the arbitrariness of $\varepsilon$, it immediately follows that $y(t) \rightarrow 0$ as $t \rightarrow 0$. This completes the proof of Theorem 4.1. 


\section{Examples}

In this section, we give some examples to illustrate the feasibility of our main results in Theorems 3.5 and 4.1 .

Example 5.1. Let

$$
\begin{aligned}
& b(t)=3, d_{1}(t)=1, \omega(t)=0.8, c_{1}(t)=5, \alpha(t)=2, \beta(t)=4+2 \sin t, \gamma(t)=1, \\
& d_{2}(t)=\exp (-0.6), h_{1}(t)=5+\sin t, \quad e(t)=30, d_{3}(t)=0.3+0.3 \sin t, c_{2}(t)=2, \\
& h_{2}(t)=2.5+0.5 \sin t, \tau_{1}=\tau_{2}=0.6
\end{aligned}
$$

In this case, system (3.2) given by Lemma 3.3 has a unique positive periodic solution $\left(x_{1}^{*}(t), x_{2}^{*}(t)\right)=(4.06,3)$. Let $\varepsilon=0.5$, it is easy to know $M_{x}=\max _{0 \leq t \leq 2 \pi}\left\{x_{i}^{*}(t)+\varepsilon, i=1,2\right\}=4.56$. By a simple calculation, we have

$$
A_{T}\left(-d_{3}(t)+\tilde{f}_{1}^{*}(t) \frac{c_{2}(t) x_{1}^{*}\left(t-\tau_{2}\right)}{\alpha(t)+\beta(t) x_{1}^{*}\left(t-\tau_{2}\right)}+\tilde{f}_{2}^{*}(t) \frac{h_{2}(t)\left(x_{2}^{*}\left(t-\tau_{2}\right)\right)^{2}}{e(t)+\left(x_{2}^{*}\left(t-\tau_{2}\right)\right)^{2}}\right)=1.12>0 .
$$

According to Theorem 3.5, system (2.5) with the above coefficients is permanent and admits at least one positive $2 \pi$-periodic solution for any nonnegative $2 \pi$-periodic function $q(t)$. Figure 1 shows the dynamic behavior of system (2.5) with the above coefficients and $q(t)=0.07$. Figure 2 shows the dynamic behavior of system

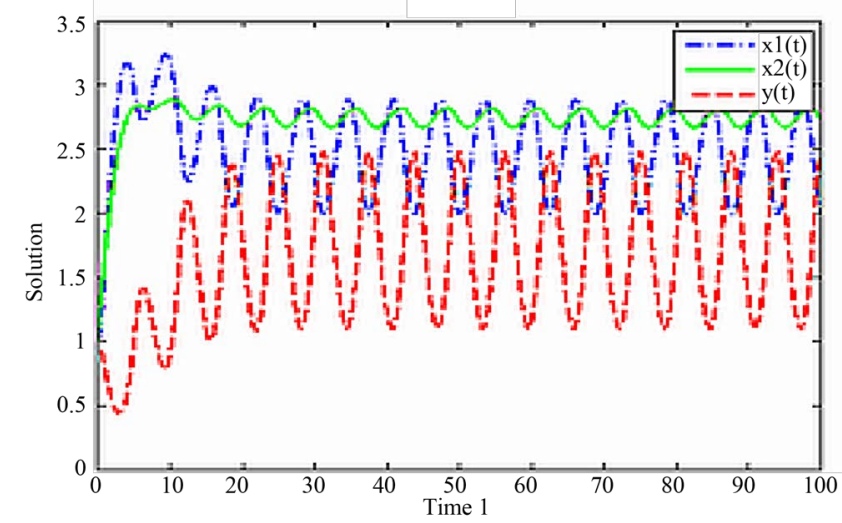

Figure 1. The periodic solution found by numerical integration of system (2.5) with initial condition $\left(\phi_{1}(\theta), \phi_{2}(\theta), \psi(\theta)\right)=(1,1,1), q(t)=0.07$ and the other parameters given by equation (5.1), here $t \in[0,100]$ and $\theta \in[-0.6,0]$.

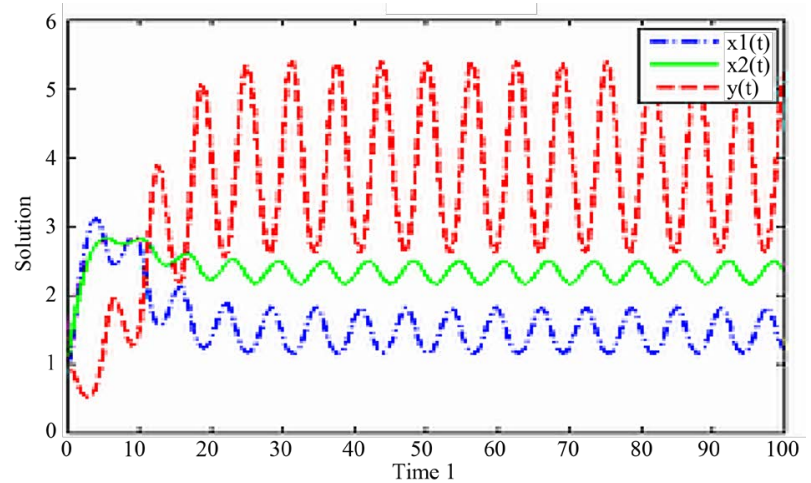

Figure 2. The periodic solution found by numerical integration of system (2.5) with initial condition $\left(\phi_{1}(\theta), \phi_{2}(\theta), \psi(\theta)\right)=(1,1,1), q(t)=0$ and the other parameters given by equation (5.1), here $t \in[0,100]$ and $\theta \in[-0.6,0]$. 
(2.5) with the above coefficients and $q(t)=0$. From Theorem 3.5, we know that the overcrowding rate $q(t)$ of the predator population has no influence on the permanence of system (2.5). However, from Figure 1 and Figure 2, we can know that the overcrowding rate influence the density of the predator population. Figure 1 demonstrates that the predator species is at low density with the overcrowding effect; Figure 2 shows that the predator population is at high density without the overcrowding effect. According to the biological viewpoint, the overcrowding effect restricts population growth.

Example 5.2. In system (2.5), let $\omega(t)=0.2, q(t)=0.07$. And the other parameters are given by equations (5.1). In the same way as Example 5.1, it is easy to verify that system (2.5) is permanent at this case. The dynamic behavior of system (2.5) in such conditions is given by Figure 3. Compared with Figure 1, we can know that the predator is at high population density if the predator prefers the immature prey to the mature, whereas it is at lower population density. According to the biological viewpoint, prey vulnerability is a major factor influencing the predator preference [34]. Compared with the mature prey, the immature is more easily captured by the predator. Then, the predator population density is high if the predator prefers the immature prey.

Example 5.3. In system (2.5), let $d_{3}(t)=0.75+0.3 \sin t, q(t)=0.07$. And the other parameters are given by equations (5.1). In this case, by a simple calculation, we obtain

$$
A_{T}\left(-d_{3}(t)+\tilde{f}_{1}^{*}(t) \frac{c_{2}(t) x_{1}^{*}\left(t-\tau_{2}\right)}{\alpha(t)+\beta(t) x_{1}^{*}\left(t-\tau_{2}\right)}+\tilde{f}_{2}^{*}(t) \frac{h_{2}(t)\left(x_{2}^{*}\left(t-\tau_{2}\right)\right)^{2}}{e(t)+\left(x_{2}^{*}\left(t-\tau_{2}\right)\right)^{2}}\right)=-0.31<0 .
$$

According to Theorem 4.1, system (2.5) is impermanent and the predator population is extinction. Numerical simulation given in Figure 4 also confirms the result.

\section{Conclusions}

In this paper, we propose and analyze a periodic predator-prey system with time delay and prey stage-structured preference by the predator. The permanence and existence of positive periodic solutions of system (2.5) are explored. The conditions for the impermanence of the system and the extinction of the predator population are obtained. By Lemma 3.3, we know that $\left(x_{1}^{*}(t), x_{2}^{*}(t)\right)$ is the globally attractive periodic solution of system (2.5) without predation. Hence, the condition (3.6) implies that system (2.5) is permanence if the death rate of the predator population is small enough. Numerical simulations not only show the consistency with the theoretical analysis but also exhibit other interesting biological phenomenon. From Example 5.1, we know that the predator population is at high density without the overcrowding effect. By Examples 5.1 and 5.2, we get that the predator's preference to the immature prey is beneficial for itself development. This is because the immature prey is

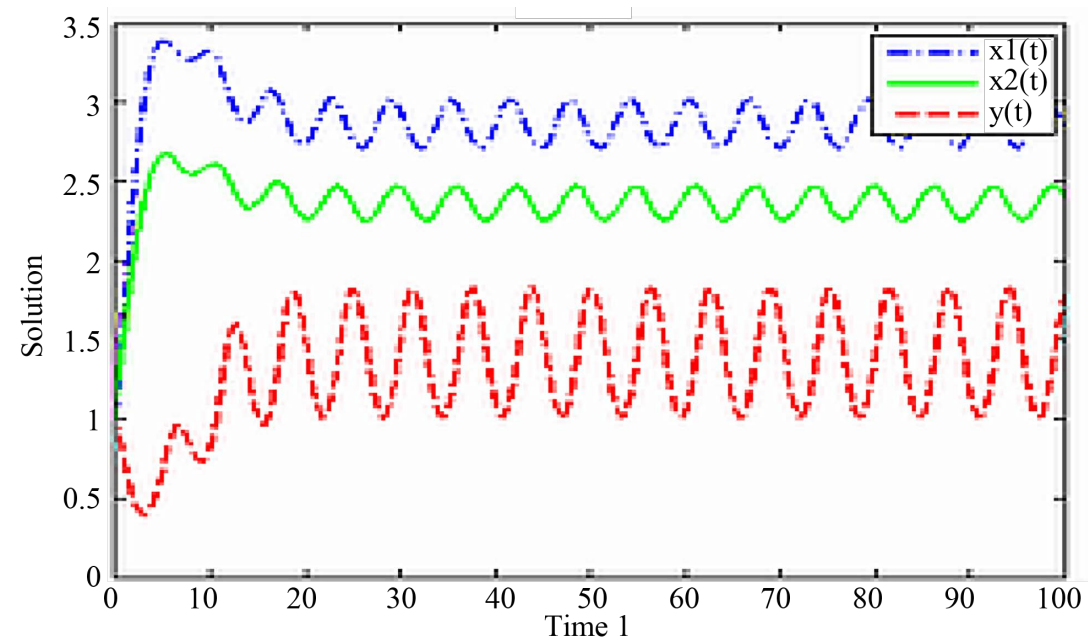

Figure 3. The periodic solution found by numerical integration of system (2.5) with initial condition $\left(\phi_{1}(\theta), \phi_{2}(\theta), \psi(\theta)\right)=(1,1,1), \omega(t)=0.2, q(t)=0.07$ and the other parameters given by equation (5.1), here $t \in[0,100]$ and $\theta \in[-0.6,0]$. 


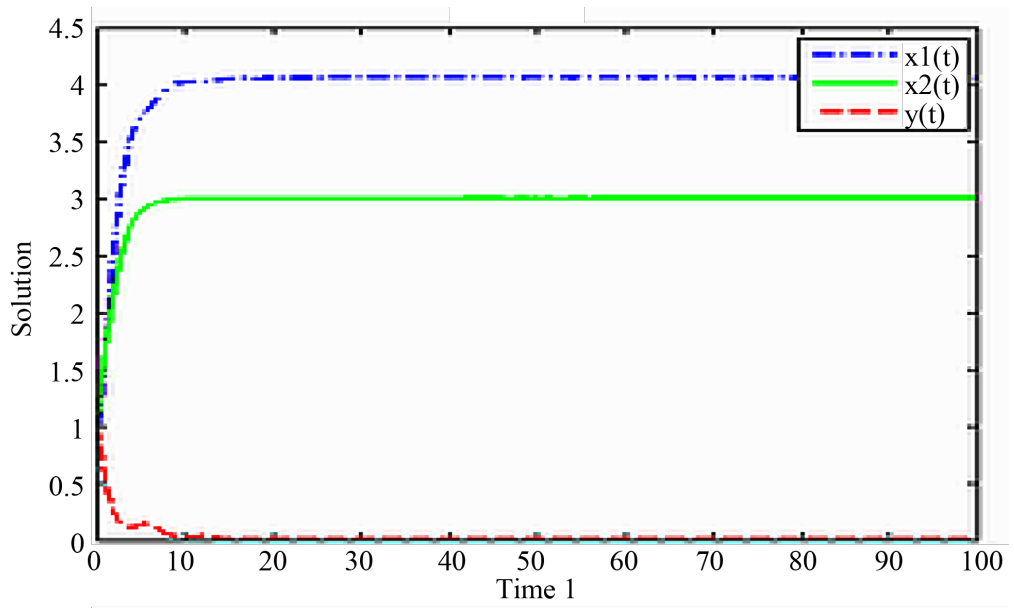

Figure 4. The temporal solution found by numerical integration of system (2.5) with initial condition $\left(\phi_{1}(\theta), \phi_{2}(\theta), \psi(\theta)\right)=(1,1,1), d_{3}(t)=0.75+0.3 \sin t$, $q(t)=0.07$ and the other parameters given by equation (5.1), here $t \in[0,100]$ and $\theta \in[-0.6,0]$.

more palatable and more easily captured by the predator than the mature. Example 5.3 illustrates the correctness of Theorem 4.1.

We would like to mention here that we are unable to solve the following questions:

1) How many positive periodic solutions exist in system (2.5)?

2) Is the solution global attractivity if system (2.5) has only one positive periodic solution?

We leave these for future work.

\section{Acknowledgements}

The authors express sincere gratitude to the anonymous referees for their helpful comments and suggestions that led to an improvement of our original manuscript.

\section{Funding}

This work was supported by the Major Project of Sichuan University of Arts and Science (Grant No. 2014Z005Z), by the General Project of Educational Commission in Sichuan Province (Grant No. 16ZB0357).

\section{References}

[1] Falconi, M., Huenchucona, M. and Vidal, C. (2015) Stability and Global Dynamic of a Stage-Structured Predator-Prey Model with Group Defense Mechanism. Applied Mathematics and Computation, 270, 47-61. http://dx.doi.org/10.1016/j.amc.2015.07.109

[2] Fawzi, J. and Omari, M. (2015) The Effect of State Dependent Delay and Harvesting on a Stage-Structured PredatorPrey Model. Applied Mathematics and Computation, 271, 142-153. http://dx.doi.org/10.1016/j.amc.2015.08.119

[3] Meng, X.-Y., Huo, H.-F., Xiang, H. and Yin, Q.-Y. (2014) Stability in a Predator-Prey Model with Crowley-Martin Function and Stage Structure for Prey. Applied Mathematics and Computation, 232, 810-819. http://dx.doi.org/10.1016/j.amc.2014.01.139

[4] Liu, C., Zhang, Q.-L. and Huang, J. (2013) Stability Analysis of a Harvested Prey-Predator Model with Stage Structure and Maturation Delay. Mathematical Problems in Engineering, 2013, Article ID: 329592. http://dx.doi.org/10.1155/2013/329592

[5] Xu, R., Chaplain, M. and Davidson, F. (2004) Persistence and Global Stability of a Ratio-Dependent Predator-Prey Model with Stage Structure. Applied Mathematics and Computation, 158, 729-744. http://dx.doi.org/10.1016/j.amc.2003.10.012

[6] Deng, L.-W., Wang, X.-D. and Peng, M. (2014) Hopf Bifurcation Analysis for a Ratio-Dependent Predator-Prey Sys- 
tem with Two Delays and Stage Structure for the Predator. Applied Mathematics and Computation, 231, 214-230. http://dx.doi.org/10.1016/j.amc.2014.01.025

[7] Qu, Y. and Wei, J. (2010) Bifurcation Analysis in a Predator-Prey System with Stage-Structure and Harvesting. Journal of the Franklin Institute, 347, 1097-1113. http://dx.doi.org/10.1016/j.jfranklin.2010.03.017

[8] Ma, Z., Wang, S., Wang, W. and Li, Z. (2011) Permanence of a Stage-Structured Predator-Prey System with a Class of Functional Responses. Comptes Rendus Biologies, 334, 851-854. http://dx.doi.org/10.1016/j.crvi.2011.08.002

[9] Zhang, L.-M. and Zhang, C.-F. (2010) Rich Dynamic of a Stage-Structured Prey-Predator Model with Cannibalism and Periodic Attacking Rate. Communications in Nonlinear Science and Numerical Simulation, 15, 4029-4040. http://dx.doi.org/10.1016/j.cnsns.2010.02.009

[10] Zhang, Z.-Q. and Luo, J. (2010) Multiple Periodic Solutions of a Delayed Predator-Prey System with Stage Structure for the Predator. Nonlinear Analysis: Real World Applications, 11, 4109-4120. http://dx.doi.org/10.1016/j.nonrwa.2010.03.015

[11] Nie, L., Teng, Z., Hu, L. and Peng, J. (2009) Existence and Stability of Periodic Solution of a Predator-Prey Model with State-Dependent Impulsive Effects. Mathematics and Computers in Simulation, 79, 2122-2134. http://dx.doi.org/10.1016/j.matcom.2008.11.015

[12] Kouche, M., Tatar, N.E. and Liu, S. (2008) Permanence and Existence of a Positive Periodic Solution to a Periodic Stage-Structured System with Infinite Delay. Applied Mathematics and Computation, 202, 620-638. http://dx.doi.org/10.1016/j.amc.2008.03.002

[13] Chen, F. and You, M. (2008) Permanence, Extinction and Periodic Solution of the Predator-Prey System with Beddington-DeAngelis Functional Response and Stage Structure for Prey. Nonlinear Analysis: Real World Applications, 9, 207-221. http://dx.doi.org/10.1016/j.nonrwa.2006.09.009

[14] Xia, Y., Cao, J. and Cheng, S. (2007) Multiple Periodic Solutions of a Delayed Stage-Structured Predator-Prey Model with Non-Monotone Functional Responses. Applied Mathematical Modelling, 31, 1947-1959. http://dx.doi.org/10.1016/j.apm.2006.08.012

[15] Chen, F.D. (2006) Permanence of Periodic Holling Type Predator-Prey System with Stage Structure for Prey. Applied Mathematics and Computation, 182, 1849-1086. http://dx.doi.org/10.1016/j.amc.2006.06.024

[16] Xu, R., Chaplain, M.-A.-J. and Davidson, F.A. (2005) Permanence and Periodicity of a Delayed Ratio-Dependent Predator-Prey Model with Stage Structure. Journal of Computational and Applied Mathematics, 303, 602-621.

[17] Bence, J.-R. and Nisbet, R.-M. (1989) Space Limited Recruitment in Open Systems: The Importance of Time Delays. Ecology, 70, 1434-1441. http://dx.doi.org/10.2307/1938202

[18] Berryman, A.-A. (1992) The Origins and Evolution of Predator-Prey Theory. Ecology, 75, 1530-1535. http://dx.doi.org/10.2307/1940005

[19] Cushing, J.-M. (1977) Periodic Time Dependent Predator-Prey System. SIAM Journal on Applied Mathematics, 32, 8295. http://dx.doi.org/10.1137/0132006

[20] Krukonis, G. and Schaffer, W.-M. (1991) Population Cycles in Mammals and Birds: Does Periodicity Scale with Body Size? Journal of Theoretical Biology, 148, 469-493. http://dx.doi.org/10.1016/S0022-5193(05)80232-2

[21] Cui, J.-A. and Song, X.-Y. (2004) Permanence of a Predator-Prey System with Stage Structure. Discrete Continuous Dynamic Systems-Series B, 4, 547-554. http://dx.doi.org/10.3934/dcdsb.2004.4.547

[22] Provost, C., Lucas, E. and Coderre, D. (2006) Prey Preference of Hyaliodes vitripennis as an Intraguild Predator: Active Predator Choice or Passive Selection? Biological Control, 37, 148-154. http://dx.doi.org/10.1016/j.biocontrol.2006.01.005

[23] Baalen, M., Krivan, V., van Rijn, P.-C. and Sabelis, M.-W. (2001) Alternative Food, Switching Predators, and the Persistence of Predator-Prey Systems. American Nature, 157, 512-524. http://dx.doi.org/10.1086/319933

[24] Goldman, J.-C. and Dennett, M.-R. (1990) Dynamics of Prey Selection by an Omnivorous Flagellate. Marine Ecology Progress Series, 59, 183-194. http://dx.doi.org/10.3354/meps059183

[25] O’Donoghue, M., Boutin, S., Krebs, C.-J., Zuleta, G. and Dennis, L. (1998) Functional Response of Coyotes and Lynx to the Snowshoe Hare Cycle. Ecology, 79, 1193-1208. http://dx.doi.org/10.2307/176736

[26] Chao, T., Peng, Z.-Q., Shen, Y.-X., Lu, B.-Q., Fu, Y.-G. and Wang, F.-G. (2006) Host Age Preference and Suitability of Asecodes hispinarum. Chinese Journal of Tropical Crops, 27, 78-80. (In Chinese)

[27] Li, J., Coudron, T.-A., Pan, W., Liu, X., Lu, Z. and Zhang, Q. (2006) Host Age Preference of Microplitis mediator (Hymenoptera: Braconidae), an Endoparasitoid of Mythimna separata (Lepidoptera: Noctuidae). Biological Control, 39, 257-261. http://dx.doi.org/10.1016/j.biocontrol.2006.09.002

[28] Venzon, M., Jansse, A. and Sabelis, M.-W. (2001) Prey Preference, Intraguild Predation and Population Dynamics of an Arthropod Food Web on Plants. Experimental and Applied Acarology, 25, 785-808. 
http://dx.doi.org/10.1023/A:1020443401985

[29] Rai, V., Anand, M. and Upadhyay, R.-K. (2007) Trophic Structure and Dynamical Complexity in Simple Ecological Models. Ecological Complexity, 4, 212-222. http://dx.doi.org/10.1016/j.ecocom.2007.06.010

[30] Zhao, X.-Q. (1991) The Qualitative Analysis of N-Species Lotka-Volterra Periodic Competition Systems. Mathematical and Computer Modelling, 15, 3-8. http://dx.doi.org/10.1016/0895-7177(91)90100-L

[31] Liu, S.-Q., Chen, L.-S. and Liu, Z.-J. (2002) Extinction and Permanence in Nonautonomous Competitive System with Stage Structure. Journal of Mathematical Analysis and Applications, 274, 667-684. http://dx.doi.org/10.1016/S0022-247X(02)00329-3

[32] Lakshmikantham, V., Matrosov, V.-M. and Sivasundaram, S. (1991) Vector Lyapunov Functions and Stability Analysis of Nonlinear Systems. Vol. 63, Kluwer Academic Publishers, Dordrecht, Boston, London. http://dx.doi.org/10.1007/978-94-015-7939-1

[33] Teng, Z. and Chen, L. (1999) The Positive Periodic Solutions in Periodic Kolmogorov Type Systems with Delays. Acta Mathematicae Applicatae Sinica, 22, 446-456.

[34] Rai, V., Anand, M. and Upadhyay, R.K. (2007) Trophic Structure and Dynamical Complexity in Simple Ecological Models. Ecological Complexity, 4, 212-222. http://dx.doi.org/10.1016/j.ecocom.2007.06.010 